\title{
Effects of firing frequency on length-dependent myofascial force transmission between antagonistic and synergistic muscle groups
}

\author{
H. J. M. Meijer · J. M. Rijkelijkhuizen • \\ P. A. Huijing
}

Accepted: 28 May 2008/Published online: 27 June 2008

(C) The Author(s) 2008

\begin{abstract}
Effects of stimulation frequency on myofascial force transmission between rat peroneal and triceps surae and antagonistic anterior crural muscles, and between extensor digitorum longus (EDL) and tibialis anterior and extensor hallucis longus (TA + EHL) muscles were investigated for lengthening of all anterior crural muscles. Muscles contracted isometrically at firing rates of 10, 20, 30 and $100 \mathrm{~Hz}$. EDL and TA + EHL were distally lengthened. Peroneal and triceps surae muscles attained a constant muscle-tendon complex length. Peroneal and triceps surae distal active force decreased significantly as a function of anterior crural muscle length, also at submaximal activation. The absolute decrease was highest for $100 \mathrm{~Hz}$ (peroneal muscles $-0.87 \mathrm{~N}$; triceps surae muscles $-0.92 \mathrm{~N}$ ), but the highest normalized decrease occurred at $10 \mathrm{~Hz}$ stimulation (peroneal muscles $-34 \%$; triceps surae muscles $-18 \%$ ). At all muscle lengths, a negative proximo-distal difference in EDL active force was present which decreased with lower firing frequencies (from $-0.4 \mathrm{~N}$ at $100 \mathrm{~Hz}$ to $-0.03 \mathrm{~N}$ at $10 \mathrm{~Hz}$ ). The passive proximo-distal force difference attained positive values. EDL and TA + EHL length-force characteristics agree with effects of firing frequency, except for $10 \mathrm{~Hz}$ stimulation, where active force was higher than
\end{abstract}

H. J. M. Meijer $(\bowtie) \cdot$ J. M. Rijkelijkhuizen · P. A. Huijing

Instituut voor Fundamentele en Klinische

Bewegingswetenschappen, Faculteit Bewegingswetenschappen,

Vrije Universiteit, Van der Boechorststraat 9,

1081 BT Amsterdam, The Netherlands

e-mail: meijerh@naturalis.nl

P. A. Huijing

Integrated Biomechanical Engineering for Restoration of Human

Function, Instituut voor Biomedische Technologie,

Faculteit Constructieve Wetenschappen, Universiteit Twente,

Enschede, The Netherlands expected and optimum length shifted to lower muscle lengths. It is concluded that also at submaximal stimulation frequencies, extramuscular myofascial force transmission between peroneal and triceps surae muscles and antagonistic anterior crural muscles is substantial. Although lengthening of submaximally active anterior crural muscles decreases the net myofascially transmitted load on EDL, myofascial force transmission significantly alters effects of firing frequency on length-force characteristics.

Keywords Myofascial force transmission .

Anterior crural compartment - Peroneal compartment .

Triceps surae compartment · Firing frequency

\begin{tabular}{|c|c|}
\hline \multicolumn{2}{|c|}{ Abbreviations } \\
\hline EDL & $\begin{array}{l}\text { Extensor digitorum longus muscle-tendon } \\
\text { complex }\end{array}$ \\
\hline $\mathrm{TA}+\mathrm{EHL}$ & $\begin{array}{l}\text { Tibialis anterior and extensor hallucis } \\
\text { longus muscle-tendon complex }\end{array}$ \\
\hline$F_{\mathrm{m}}$ & $\begin{array}{l}\text { Total force (measured during tetanic } \\
\text { contraction) }\end{array}$ \\
\hline$F_{\mathrm{mp}}$ & $\begin{array}{l}\text { Passive force (measured prior to tetanic } \\
\text { contraction) }\end{array}$ \\
\hline$F_{\mathrm{ma}}$ & $\begin{array}{l}\text { Active force, calculated by subtracting } F_{\mathrm{mp}} \\
\text { from } F_{\mathrm{m}}\end{array}$ \\
\hline$\Delta l_{m+\mathrm{t}}$ & $\begin{array}{l}\text { Length of the muscle-tendon complex } \\
\text { expressed as a deviation of optimum } \\
\text { muscle length }\end{array}$ \\
\hline$l_{\text {mao }}$ & $\begin{array}{l}\text { Optimum muscle length, i.e. muscle length } \\
\text { which shows maximum active force }\end{array}$ \\
\hline$F_{\text {mao }}$ & Maximum active force \\
\hline$\Delta F_{\mathrm{m}}$ & $\begin{array}{l}\text { The difference in total force exerted at the } \\
\text { proximal and distal EDL tendon, calculated } \\
\text { by subtracting proximal total force from } \\
\text { distal total force }\end{array}$ \\
\hline
\end{tabular}


$\Delta F_{\mathrm{ma}} \quad$ The difference in active force exerted at the proximal and distal EDL tendon, calculated by subtracting proximal active force from distal active force

$\Delta F_{\mathrm{mp}} \quad$ The difference in passive force exerted at the proximal and distal EDL tendon, calculated by subtracting proximal passive force from distal passive force

\section{Introduction}

In recent years, it has been shown that muscle force is not only transmitted via tendinous connections (Tidball (1991) on the frog semitendinous, and Trotter et al. (1985) on mouse gastrocnemius muscle) but also via transsarcolemmal pathways of the costameres (i.e. integrin and the dystroglycan complex (Monti et al. 1999; Pasternak et al. 1995; Sheard et al. 2002)).This means that the cytoskeleton is connected to connective tissue structures within and surrounding the muscle and force transmission will take place between these structures (i.e. myofascial force transmission, (Huijing and Baan 2001a; Huijing et al. 1998; Maas et al. 2001; Yucesoy et al. 2003)).

Epimuscular myofascial force transmission (i.e. transmission trough the epimysium to other muscles or nonmuscular tissues) is indicated by a difference in force exerted at the origin and insertion of a muscle. This principle was demonstrated by Huijing and Baan (2001a), who showed that for rat extensor digitorum muscle (EDL) within an intact network of connective tissue, forces measured at the proximal tendon were usually not equal to force measured at the distal tendons. Via myofascial connections, a fraction of the muscle force can be transmitted from one muscle onto another, without passing one of its tendons. Therefore, any difference in force measured at the origin and insertion of a muscle is an indication of myofascial force transmission between a muscle and its surrounding tissues. An important consequence of myofascial force transmission is that sarcomeres are not only loaded by forces transmitted via the myotendinous pathways and sarcomeres in series, but also by forces transmitted by myofascial connections. The direction and magnitude of these myofascially transmitted forces has been found to depend upon muscle length as well as the muscle's position relative to its surroundings (Huijing and Baan 2003; Maas et al. 2003a, b; Yucesoy et al. 2006). At low firing frequencies, which are common for in vivo muscle functioning (Hennig and Lomo 1985), myofascial force transmission between synergistic muscles was found to be relatively more important (Meijer et al. 2006).
In recent years, it has become clear that myofascial force transmission between muscles is not limited to synergistic muscles, but also occurs between antagonistic muscles, (Huijing 2007; Huijing and Jaspers 2005; Rijkelijkhuizen et al. 2007). Meijer et al. (2007) showed that lengthening of a whole group of maximally active synergistic rat muscles significantly affects the force exerted by antagonistic muscles. Key in understanding such interaction is the fact that the transmission of muscle force via myofascial pathways is inherent to the integration of muscles within a higher level of tissue organization. Therefore, any muscle in vivo may be affected by myofascial force transmission, and considering myofascial structures as co-determinants of muscle force has already proven to be successful in human tendon transfer surgery (Smeulders and Kreulen 2007).

To enhance understanding of the physiological relevance of myofascial interactions between antagonists, it is important to assess the effects of submaximal firing frequencies on extramuscular myofascial force transmission between antagonistic muscles. Therefore, main aims of this study are: 1 . to quantify effects of stimulation frequency on myofascial force transmission between antagonistic and synergistic muscles of the rat lower hind leg at different lengths of anterior crural muscles and 2. test the hypothesis that anterior crural muscle length-force curves are affected by such transmission at different firing rates. Note that the use of a rat experimental model in the current experiment allows for greater precision and control of experimental conditions than will ever be possible in humans.

\section{Methods}

Surgical and experimental procedures were in agreement with the guidelines and regulations concerning animal welfare and experimentation set forth by Dutch law, and approved by the Committee on Ethics of Animal Experimentation at the Vrije Universiteit. Immediately after all experiments, animals were killed using an overdose of urethane solution, and double-sided pneumothorax was performed.

\section{Surgical procedures}

Male Wistar rats $(n=6$, with mean body mass of $302.6 \mathrm{~g}$ (SD 16.2)) were anaesthetized using intraperitoneally injected urethane solution $\left(1.5 \mathrm{~g} \mathrm{~kg}^{-1}\right.$ body mass, $12.5 \%$ urethane solution). Extra doses were given if necessary (maximally $1.5 \mathrm{ml}$ ). During surgery and data collection, the animals were placed on a heated water pad of approximately $37^{\circ} \mathrm{C}$ to prevent hypothermia. The skin and the biceps femoris muscle of the left hind limb were 
removed, exposing the anterior crural compartment containing the extensor digitorum longus (EDL), extensor hallucis longus (EHL) and tibialis anterior (TA) muscles. Proximal, as well as distal EDL forces are measured because differences between those forces is proof for epimuscular myofascial force transmission. In other muscles studied this is not possible since their muscle fibers originate from dispersed areas of attachment on bone and connective tissues, rather than from a proximal tendon.

As connective tissue near the muscle bellies within the anterior crural and peroneal compartments is a pathway for force transmission, this tissue was left fully intact, and limited fasciotomy was performed exclusively in the foot to severe the transverse crural ligament and the crural cruciate ligament in order to be able to free the distal tendons of EDL, EHL and TA. The original position of the TA + EHL muscles relative to the EDL in the reference position (i.e. corresponding to a knee angle of $110^{\circ}$ and ankle angle of $180^{\circ}$ plantar flexion) was recorded by placing aligned markers on the distal TA, EHL and EDL tendons. The position of the proximal EDL tendon in the reference position was marked with a small pin on the epicondylus lateralis of the femur. The proximal EDL tendon was freed by cutting a small piece of the bone with the origin of the EDL muscle. The four distal tendons of EDL were tied together at the reference position using polyester thread. After tying these tendons, they were severed distally to the knot.

The distal tendons of TA and EHL muscles were tied together and will from here on be referred to as TA + EHL. Subsequently, the distal tendons of triceps surae were severed, as well as the distal tendons of the peroneal muscles. These two muscle-tendon complexes will be referred to as triceps surae complex and peroneal complex respectively. All four distal muscle-tendon complexes (i.e. distal EDL, TA + EHL, triceps surae complex and peroneal complex), as well as the proximal EDL tendon were connected to metal rods using $100 \%$ polyester yarn. The sciatic nerve was dissected free and severed as proximally as possible. The foot was firmly fixed to a plastic plate.

\section{Mounting the animal in the experimental set up}

The rat was placed on a heated platform $\left(37^{\circ} \mathrm{C}\right)$ to prevent hypothermia. The femur was clamped to ensure a knee angle of $110^{\circ}$. The foot, attached to the plate, was firmly fixed into a rigid frame with the ankle in extreme plantar flexion $\left(180^{\circ}\right)$. Before each experiment, all force transducers (BLH Electronics Inc., Canton MA, compliance $16.2 \mu \mathrm{m} \mathrm{N}^{-1}$, mounted on single-axis micropositioners) to be used in the experiments were calibrated within the experimental setup. To eliminate differences between the force transducers, the proximal force transducer for EDL was calibrated, i.e. placed in a vertical position and calibrated by the use of weights. Subsequently, the proximal force transducer was placed back in its original horizontal position and the distal force transducer for EDL was calibrated against the proximal force transducer, while being attached to the proximal one via a compliant spring. The force transducers for TA + EHL, the triceps surae complex and peroneal complex were calibrated against the proximal force transducer in a similar fashion. All tendons were connected to the force transducers by metal rods. The metal rods of triceps surae and peroneal muscles passed dorsally over the foot, whereas the metal rods of TA + EHL and distal EDL passed ventrally under the foot (Fig. 1b). All metal rods were visually aligned with the muscles' line of pull. The sciatic nerve was placed on a pair of silver electrodes and prevented from dehydration by covering it with paper tissue (saturated with isotonic saline) and by a thin piece of latex.

\section{Experimental conditions}

Ambient temperature $\left(22^{\circ} \mathrm{C} \pm 0.5\right)$ and air humidity $(70 \pm 2 \%)$ were kept constant by a computer-controlled air-conditioning system (Holland Heating, Waalwijk, the Netherlands). Muscle and tendon tissue was further prevented from dehydration by regular irrigation with isotonic saline. The peroneal complex and triceps surae muscles were kept at a constant muscle-tendon length, set to exert an initial force of 5 and $11 \mathrm{~N}$ respectively. At these muscle lengths, passive forces were very small. Note that the initial values of peroneal and triceps surae active forces in Figs. 2 and 3 are lower than the forces set at the start of the experiment. These initial force levels were determined with all other muscles kept at their respective active slack length, which is defined as the low muscle length at which active force exerted approaches zero. Note that lengthening of surrounding muscles at their distal tendons will increase the myofascial load on the peroneal and triceps surae muscles and will decrease the force exerted at the distal tendons of the muscle considered. The position of the proximal EDL tendon was set to correspond to a length that was $2 \mathrm{~mm}$ shorter than the original marker position (as judged from the the marker on the femur). The original position of TA + EHL relative to the EDL in the reference position was preserved by aligning the markers on the distal TA, EHL and EDL tendons. This relative position was maintained during the experiment by moving EDL and $\mathrm{TA}+\mathrm{EHL}$ distal force transducers equal distances.

Prior to excitation, all muscles were brought to their desired length and position passively by moving the force transducers. Before acquiring data, EDL and TA + EHL were preconditioned by isometric contractions at alternating high and low lengths, until forces at low length were 


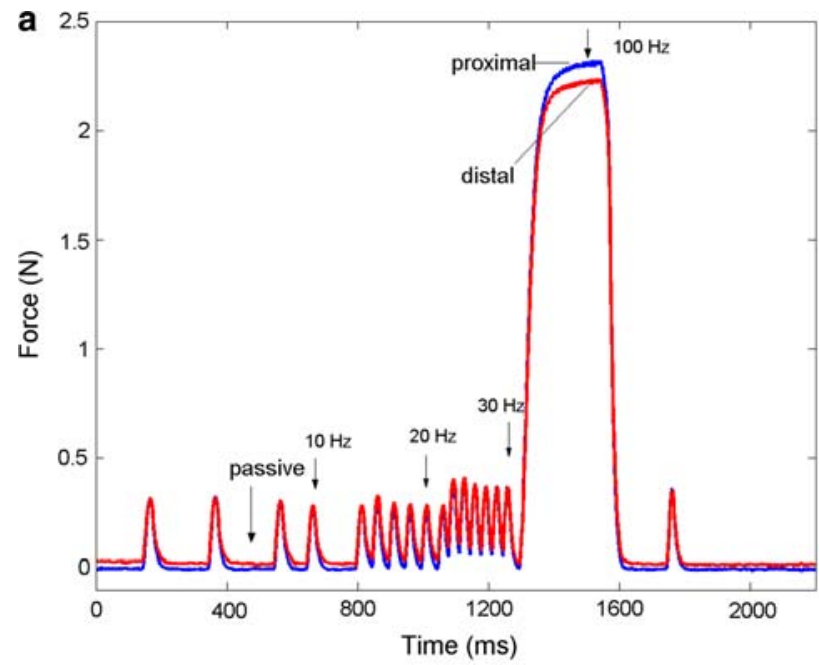

b

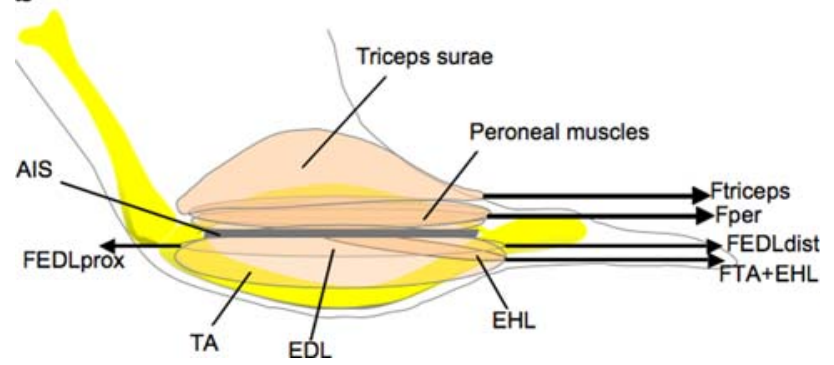

Fig. 1 a Example of proximal as well as distal time-force traces of EDL muscle. An ascending stimulation frequency protocol of 10, 20, 30 and $100 \mathrm{~Hz}$ was applied to the nerve innervating the muscle. The example shows results for a contraction near optimum length (i.e. length at which the muscle exerts maximum active force). Arrows indicate the instants at which active $\left(F_{\mathrm{ma}}\right)$ and passive $\left(F_{\mathrm{mp}}\right)$ force data are measured for composing length-force curves. b Schematic view of the experimental set up of the rat lower leg. Lateral view of the rat anterior crural, peroneal and triceps surae compartments after removal of the skin and $\mathrm{m}$. biceps femoris. Connective tissues at the muscle bellies were left intact. The $\mathrm{m}$. tibialis anterior and $\mathrm{m}$. extensor hallucis longus complex (TA + EHL) and $\mathrm{m}$. triceps surae muscles are shown transparent to allow showing of the position of the underlying $\mathrm{m}$. extensor digitorum longus (EDL), $\mathrm{m}$. extensor hallucis longus (EHL) and peroneal muscles (PER). Note that EHL originates at the anterior intermuscular septum (AIS). The proximal origin of the extensor digitorum longus (EDL) as well as the distal tendons of the triceps surae, peroneal, EDL and TA + EHL muscles were severed and connected to force transducers using metal rods. The forces measured are represented by arrows. Both tibia and foot were fixed within a rigid frame in such a way that the muscles were aligned with the force transducers

reproducible (i.e. effects of previous activity at high length (Huijing and Baan 2001a) are removed). For the lengthforce curve, TA + EHL and EDL muscles were lengthened distally with $1 \mathrm{~mm}$ increments and isometric contractions were performed at different EDL and TA + EHL lengths. All muscles studied were stimulated through the sciatic nerve with a constant current $(3 \mathrm{~mA})$. The stimulation protocol started with two twitches (at $t=200$ and $t=400 \mathrm{~ms})$, followed by a pulse train (at $t=600 \mathrm{~ms})$ with a Fma Peroneal complex (N)

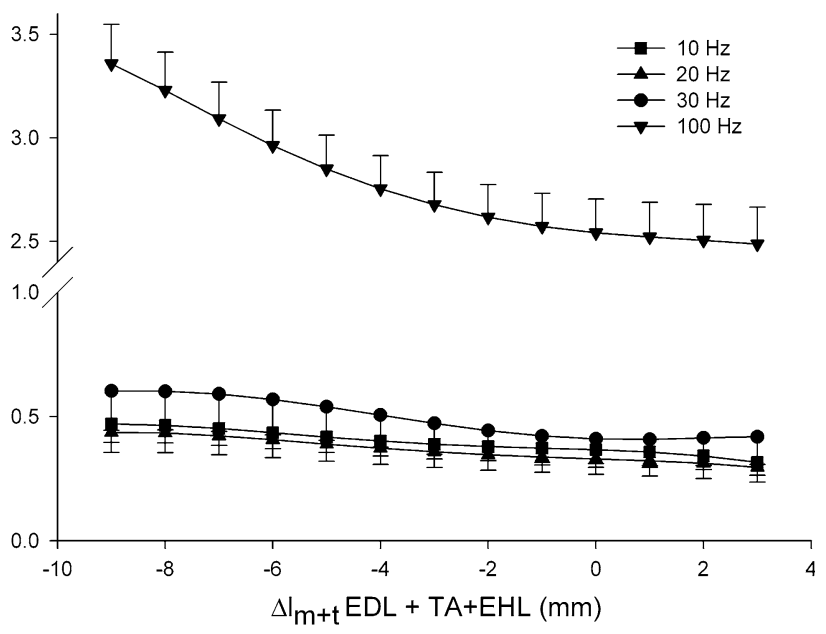

b Fma Peroneal complex (\%Fma initial)

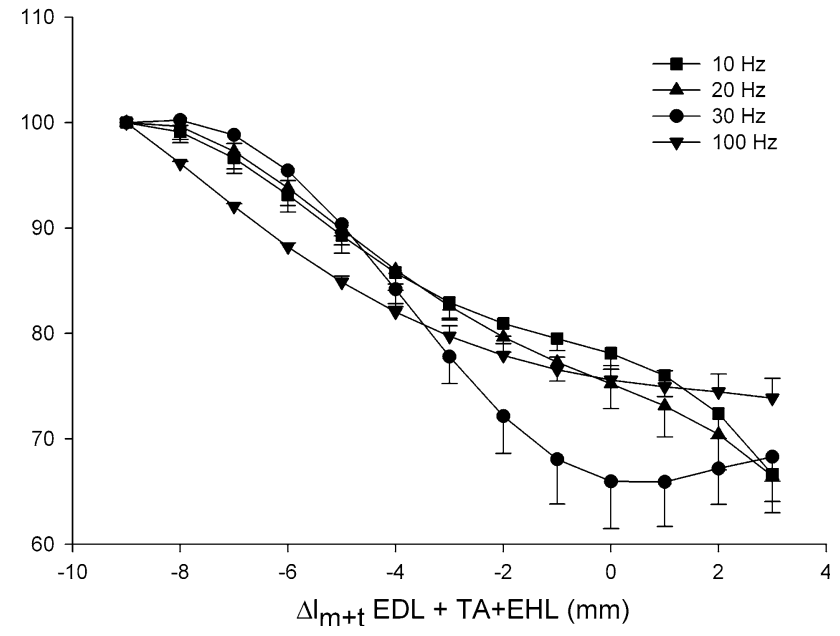

Fig. 2 Effects of stimulation frequency on forces exerted distally by peroneal muscles, which were kept at a constant length. a Peroneal active forces $\left(F_{\text {ma }}\right)$ during the tetanic plateau at $10,20,30$ and $100 \mathrm{~Hz}$ stimulation as a function of lengths of the anterior crural muscles. b Peroneal active forces $\left(F_{\mathrm{ma}}\right)$, normalized for initial force level per frequency, as a function of lengths of anterior crural muscles. Anterior crural muscle-tendon complex $\left(\Delta l_{m+t}\right)$ length is expressed as a deviation from distal EDL $100 \mathrm{~Hz}$ optimum length (i.e. length at which the muscle exerts maximum active force at $100 \mathrm{~Hz}$ firing frequency). Values are shown as mean $\pm \mathrm{SE}, n=6$

a staircase complex of ascending stimulation frequencies of 10,20 , and 30 and $100 \mathrm{~Hz}$ (pulse width $0.1 \mathrm{~ms}$, total stimulation time $1,000 \mathrm{~ms}$ ), and a final twitch at $t=1,800 \mathrm{~ms}$. Timing of stimulation of the nerve and A/D conversion (12-bit $\mathrm{A} / \mathrm{D}$ converter, sampling frequency $1,000 \mathrm{~Hz}$, resolution of force $0.01 \mathrm{~N}$ ) were controlled by a special purpose microcomputer. After each contraction, the muscles were allowed to recover near active slack length for 2 min. Passive isometric force was measured prior to the tetanic contraction and total force was measured during the tetanic plateau of the muscle force (see Fig. 1a). 
a Fma Triceps (N)

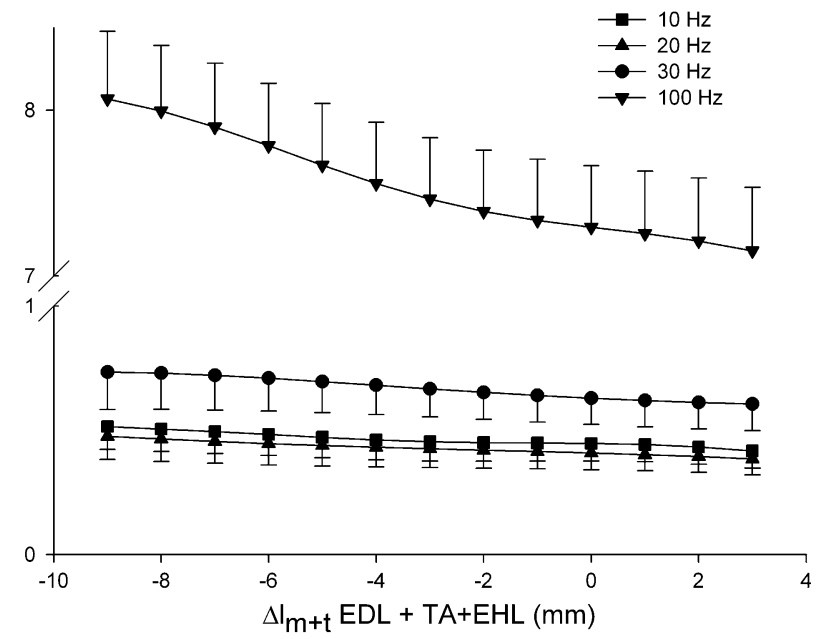

b Fma Triceps (\%Fma initial)

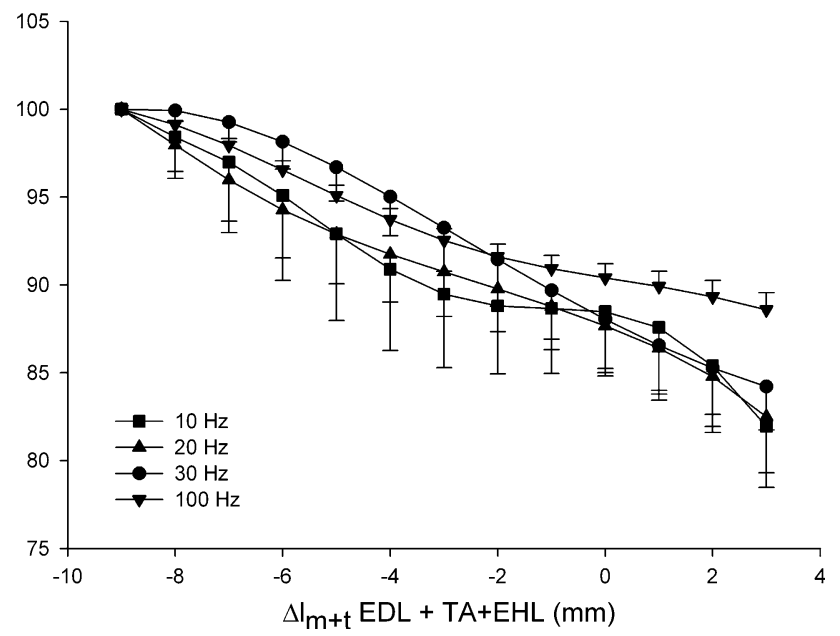

Fig. 3 Effects of stimulation frequency on forces exerted distally by triceps surae muscles which were kept at a constant length. a Triceps surae active forces $\left(F_{\mathrm{ma}}\right)$ during the tetanic plateau at firing rates of $10,20,30$ and $100 \mathrm{~Hz}$ as a function of the length of anterior crural muscles. b Triceps surae active forces $\left(F_{\text {ma }}\right)$, normalized for initial force level per frequency, as a function of the length of anterior crural muscles. Lengthening of the anterior crural muscles was performed by moving the distal force transducers of EDL and TA + EHL only, and anterior crural muscle-tendon complex length $\left(\Delta l_{m+t}\right)$ is expressed as a deviation from distal EDL $100 \mathrm{~Hz}$ optimum length (i.e. length at which the muscle exerts maximum active force at $100 \mathrm{~Hz}$ firing frequency). Values are shown as mean $\pm \operatorname{SE}(n=6)$

Data treatment

Data relating passive muscle force to length was fitted by an exponential curve using a least-squares criterion:

$y=\exp (a x+b)+C$

where $y$ represents passive muscle force, $x$ represents muscle-tendon complex length and " $a$ ", " $b$ " and " $C$ " are fitting constants. Active muscle force $\left(F_{\mathrm{ma}}\right)$ was estimated by subtracting the calculated passive force $\left(F_{\mathrm{mp}}\right)$ using the fitted function, from total force $\left(F_{\mathrm{m}}\right)$ for the appropriate muscle length.

Active length-force data were then fitted with a stepwise polynomial regression procedure. In this fitting procedure, the curve fitting starts with a first order polynomial and the power is increased up to a sixth order, as long as this yields a significant improvement to the description of the lengthactive force data (see statistics). The polynomial used for fitting active length-force data is the following:

$y=b_{0}+b_{1} x+b_{2} x^{2}+\cdots b_{n} x^{n}$

where $y$ represents active muscle force, $x$ represents active muscle force length and $b_{0}$ through $b_{n}$ are fitting constants. Using the selected polynomials, mean and standard errors of active muscle force were calculated for given muscle lengths. Optimum muscle length was defined for each individual curve as the active muscle length at which the fitted active force curve showed maximum force $\left(F_{\text {mao }}\right)$. Active slack length for TA + EHL and distal EDL was estimated by selecting data of muscle length and active muscle force $\left(F_{\text {ma }}<0.3 x F_{\text {mao }}\right)$ and extrapolated with a fitted curve where

$y=\exp \left(b_{0} x+b_{1}\right)+b_{2}$.

Active slack length was determined by solving the roots for this equation and is plotted with horizontal error bars.

For EDL, the differences in total, passive and active force between the distal and proximal tendon (respectively $\Delta F_{\mathrm{m}}, \Delta F_{\mathrm{mp}}$ and $\Delta F_{\mathrm{ma}}$ ) were calculated by subtracting proximal force from distal force. The normalized active force difference was calculated for each frequency by expressing the absolute active force difference as a percentage of the optimum distal active force for that frequency. Normalized peroneal and triceps surae active forces were calculated by expressing active forces as a percentage of the initial active force per frequency.

Definitions of myofascial force transmission via different pathways

Three types of myofascial force transmission are distinguished. 1. Muscle force generated by the sarcomeres can be transmitted onto the basal lamina and the endomysium (Bloch and Gonzalez-Serratos 2003; Monti et al. 1999; Street 1983; Street and Ramsey 1965; Sheard et al. 2002). From that point, the intramuscular stroma of connective tissue enables the transmission of force onto the perimysium as well as the epimysium. This is referred to as intramuscular myofascial force transmission (Huijing 2003). 2. For muscles within their natural context of connective tissue, the continuity of intramuscular stromata of adjacent muscles creates pathways for the transmission of force between these muscles. Such transmission is referred 
to as intermuscular myofascial force transmission (Huijing and Baan 2001b; Maas et al. 2001). Third, when force is transmitted between the epimysium of a muscle and a nonmuscular structure, such as connective tissues reinforcing nerves and blood vessels, intermuscular septa, the interosseal membrane, compartmental fascia and epitendinous tissues (Rijkelijkhuizen et al. 2005), it is referred to as extramuscular myofascial force transmission (Huijing and Baan 2001a). Note that when muscle force is transmitted via the epimysium, it is generally referred to as epimuscular myofascial force transmission until further distinction of pathways is possible.

\section{Statistics}

In the active muscle force fitting procedure, the curve fitting starts with a first order polynomial and the power was increased up to a sixth order, as long as this yields a significant improvement to the description of the lengthactive force data, as determined by one-way analysis of variance (ANOVA) (Neter et al. 1990). Two-way ANOVA's were used to test for the effects of anterior crural muscle length and firing frequency on absolute and normalized peroneal and triceps surae active forces. In addition, two-way ANOVA's were used to test for the effects of stimulation frequency and anterior crural muscle length on EDL proximal and distal active and passive forces, TA + EHL active forces, the EDL proximo-distal active force differences as well as proximally and distally measured optimum muscle length $\left(L_{\text {mao }}\right)$. One-way ANOVA's were used to test for the effects of firing frequency on distal EDL and distal TA + EHL active slack lengths and to test for the effects of anterior crural muscle length on $\mathrm{TA}+\mathrm{EHL}$ passive force and the EDL proximo-distal difference in passive forces. A one sample $t$ test was used to test whether EDL $\Delta F_{\text {ma }}$ was significantly different from zero. If significant effects were found, Bonferroni post-hoc tests for multiple pair wise comparisons were used to locate differences. Main and interaction effects were considered significant at $P<0.05$.

\section{Results}

Mechanical interaction between antagonistic muscle groups

\section{Effects of stimulation frequency and anterior crural muscle length on peroneal active forces}

Figure 2 shows effects of stimulation frequency on peroneal active forces as a function of anterior crural muscle length. Note that the peroneal complex has an antagonistic function with respect to the anterior crural muscles; and is separated from them by the anterior intermuscular septum. Passive forces were small $\left(F_{\mathrm{mp}}<0.04 \mathrm{~N}\right)$ and therefore not plotted. ANOVA showed significant effects of anterior crural muscle length as well as stimulation frequency on peroneal active forces, but no interaction effect. Peroneal active force increased with higher firing frequency. However, at each firing frequency, despite the fact that the peroneal complex was kept at a constant muscle-tendon complex length throughout the experiment, peroneal active force decreased as a function of anterior crural muscle length (Fig. 2a). The absolute decrease in peroneal active force is highest for $100 \mathrm{~Hz}$ stimulation $\left(\Delta F_{\max }=0.87 \mathrm{~N}\right)$. The smallest decrease is found for $20 \mathrm{~Hz}$ stimulation $\left(\Delta F_{\max }=0.14 \mathrm{~N}\right)$.

For peroneal forces, expressed as percentage of the initial force for each frequency (i.e. normalized peroneal active force, Fig. 2b), ANOVA showed significant effects of stimulation frequency and anterior crural muscle length, as well as interaction. Normalized peroneal active forces decreased as a function of anterior crural muscletendon complex length. The decrease in normalized peroneal force occurs in three phases: 1 . for $\Delta l_{m+\mathrm{t}} \leq-4$, normalized peroneal force at $100 \mathrm{~Hz}$ stimulation decreases more than at the submaximal stimulation frequencies; 2 . for $-3 \leq \Delta l_{m+t}$, this patterns changes and submaximal stimulation result in a similar $(10-20 \mathrm{~Hz})$ or even higher (30 Hz) deviation of initial peroneal force than for $100 \mathrm{~Hz}$; 3. ultimately, at $\Delta l_{m+t}=+3$, the peak decreases for 10 and $20 \mathrm{~Hz}$ stimulation (approx. 34\%) are similar to that of $30 \mathrm{~Hz}$ stimulation, but higher than for $100 \mathrm{~Hz}$ $\left(\Delta F_{\mathrm{ma}}=\right.$ approx. $\left.26 \%\right)$.

These results show that distal lengthening of anterior crural muscles significantly affects the distal active force of the antagonistic peroneal complex. For submaximal stimulation frequencies, the fraction of force transmitted this way is similar or larger at certain muscle lengths than for maximally active muscle. It is concluded that muscle-tendon complex length of synergistic muscle is a codeterminant of active force exerted by adjacent antagonistic muscles.

Effects of stimulation frequency and anterior crural muscle length on triceps surae active forces

The effects of stimulation frequency on triceps surae active forces are plotted as a function of anterior crural muscle length (Fig. 3). Passive forces were small $\left(F_{\mathrm{mp}}<0.05 \mathrm{~N}\right)$ and therefore not shown. ANOVA showed significant effects of stimulation frequency, as well as anterior crural muscle length on triceps surae active forces (Fig. 3a), but no interaction was shown. Triceps surae active force increased significantly with the ascending stimulation 
frequencies. However, for each stimulation frequency, triceps surae active force decreased significantly with distal lengthening of anterior crural muscles, despite a constant triceps surae muscle-tendon complex length. The decrease in active force is highest for $100 \mathrm{~Hz}$ stimulation $(0.92 \mathrm{~N})$. The smallest decrease in triceps surae active force is found for $20 \mathrm{~Hz}$ stimulation $(0.09 \mathrm{~N})$.

For normalized triceps surae active force (Fig. 3b), ANOVA showed significant effects of anterior crural muscle length and stimulation frequency, but no interaction effect was shown. For each stimulation frequency, normalized triceps surae active forces decreased as all anterior crural muscles were lengthened distally. The decrease in normalized triceps surae forces with increasing anterior crural muscle-tendon complex length shows two patterns; (1) at low muscle lengths $\left(\Delta l_{m+t} \leq-2\right)$, the curve for $100 \mathrm{~Hz}$ normalized triceps surae force is intermediate between the submaximal firing frequencies; (2) for higher muscle lengths, $\Delta l_{m+t} \geq-1$, the decrease in normalized force is highest for submaximal firing frequencies. Ultimately, 10 and $20 \mathrm{~Hz}$ stimulation yield the highest decrease $\left(\Delta F_{\mathrm{ma}}=\right.$ approx. $\left.18 \%\right)$, while $100 \mathrm{~Hz}$ stimulation shows the smallest decrease $\left(\Delta F_{\mathrm{ma}}=11 \%\right)$. This pattern differs from that seen for normalized peroneal force.

It is concluded that, for the experimental conditions imposed, even distant antagonistic muscles within the same segment are not independent units: distal lengthening of the anterior crural muscles significantly affects antagonistic muscles, even if they are located on the other side of the leg. Depending on length, the fraction of force transmitted myofascially is similar to that in maximally activated muscles, or even higher at some submaximal stimulation frequencies.

Effects of stimulation frequency on length-force characteristics

$$
T A+E H L
$$

ANOVA showed significant main effects of anterior crural muscle length and firing frequency on TA + EHL active forces (Fig. 4), as well as an interaction effect. Passive forces showed a length-dependent increase $(P<0.05)$.

In general, higher firing frequencies cause significantly higher active forces, and this force increase is quite lengthdependent. TA + EHL $100 \mathrm{~Hz}$ optimum length occurs at significantly lower muscle lengths compared to 20 and $30 \mathrm{~Hz}$ stimulation (ANOVA).

In contrast, results for $10 \mathrm{~Hz}$ showed some aspects that deviate very much from expectations based on intrinsic mechanisms: (a) $10 \mathrm{~Hz}$ optimum length could not be shown to differ significantly from that of the other stimulation frequencies, and thus did not fit the expected pattern
$\mathrm{Fm} T A+E H L(N)$

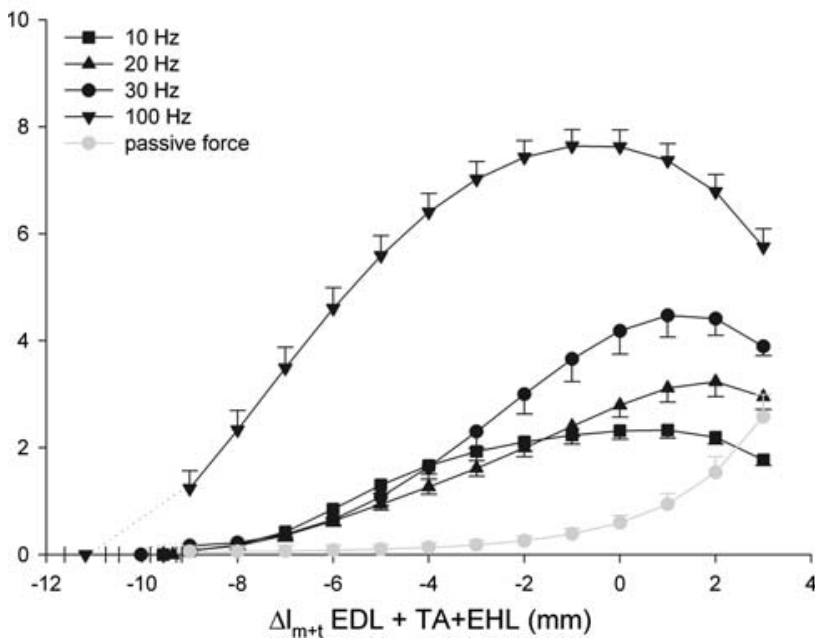

Fig. 4 Effects of stimulation frequency on TA + EHL length-force characteristics. TA + EHL passive forces $\left(F_{\mathrm{mp}}\right.$, measured before the tetanic contraction) and active forces $\left(F_{\mathrm{ma}}\right.$, calculated by subtracting passive force from total force measured during the tetanic contraction) at 10,20,30 and $100 \mathrm{~Hz}$ stimulation as a function of length of the anterior crural muscles. Lengthening of the anterior crural muscles was performed by moving the distal force transducers of EDL and TA + EHL only, and anterior crural muscle-tendon complex length $\left(\Delta l_{m+t}\right)$ is expressed as a deviation distal EDL $100 \mathrm{~Hz}$ optimum length (i.e. length at which the muscle exerts maximum active force at $100 \mathrm{~Hz}$ firing frequency). Distal active slack (low muscle length at which active force approaches zero) length was estimated by extrapolation of active muscle force to zero and displays horizontal standard errors (SE). All values are shown as mean $\pm \operatorname{SE}(n=6)$

of decreasing optimum lengths with increasing firing frequencies. (b) For the length range $-7<\Delta l_{m+t}<-2$, $10 \mathrm{~Hz}$ active forces were actually higher or equal to $20 \mathrm{~Hz}$ forces. These two results combined indicate that $\mathrm{TA}+\mathrm{EHL}$ forces are much increased with respect to expectations over a considerable length range (possibly range $-7<\Delta l_{m+t}>+2$ ).

Active slack length was also shown to significantly shift to lower muscle lengths with increasing stimulation frequency. Bonferroni post-hoc tests showed significant differences in active slack length between $100 \mathrm{~Hz}$ and the three submaximal firing frequencies, but not within the submaximal firing frequencies.

\section{$E D L$}

Figure 5a shows length-force curves for proximal and distal EDL forces at 10, 20, 30 and $100 \mathrm{~Hz}$ stimulation. For both distal and proximal active forces, ANOVA showed significant effects of anterior crural muscle length and stimulation frequency, as well as interaction between these two factors. For high lengths $\left(\Delta l_{m+t}>-2\right)$, EDL proximal and distal active forces increased at higher stimulation frequency, and this force increase is length-dependent $(P<0.05)$. 


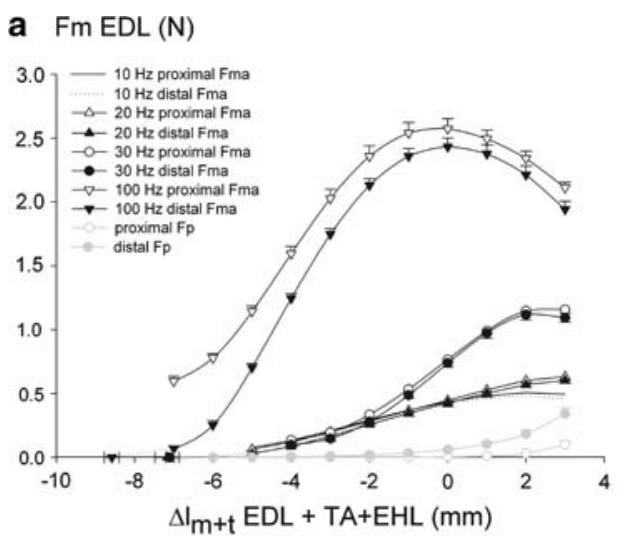

C $\Delta \mathrm{Fma}_{(\text {dist-prox) }} \& \Delta \mathrm{Fmp}$ (dist-prox) $\mathrm{EDL}(\mathrm{N})$

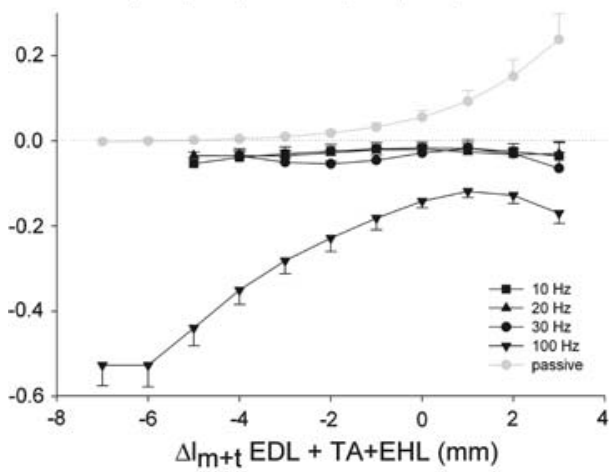

Fig. 5 EDL proximal and distal length-force characteristics and proximo-distal force differences. a EDL passive forces $\left(F_{\mathrm{mp}}\right.$, measured before the tetanic contraction) and active forces $\left(F_{\text {ma }}\right.$, calculated by subtracting passive force from total force measured during the tetanic contraction) at $10,20,30$ and $100 \mathrm{~Hz}$ firing frequencies, plotted as a function of distal length of the anterior crural muscles (EDL + TA + EHL), expressed as a deviation from distal EDL $100 \mathrm{~Hz}$ optimum length (i.e. length at which the muscle exerts maximum active force at $100 \mathrm{~Hz}$ firing frequency). Values are shown as mean $\pm \mathrm{SE}, n=6$. $\mathbf{b}$ The difference in proximally and distally

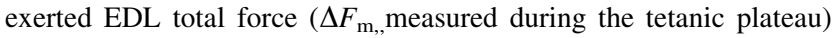
plotted as a function of anterior crural muscle length (expressed as a deviation from distal EDL $100 \mathrm{~Hz}$ optimum length [i.e. length at which the muscle exerts maximum active force at $100 \mathrm{~Hz}$ firing frequency]). The proximo-distal difference in total force $\left(\Delta F_{\mathrm{m}}\right)$ was

For 10, 30 and $100 \mathrm{~Hz}$, ANOVA showed that proximal and distal EDL active forces differ significantly and proximal active force is higher than distal active force at all muscle lengths. In contrast, for $20 \mathrm{~Hz}$, ANOVA showed no significant effect of location of force measurement (i.e. at the proximal or distal tendon) on EDL active forces. For $100 \mathrm{~Hz}$, ANOVA showed an interaction between effects of location of force measurement and length, indicating that proximal and distal EDL active forces vary in an individual way as a function of anterior crural muscle length.

Note that EDL optimum lengths measured at the proximal tendon did not differ significantly from optimum lengths measured at the distal tendon. As expected, for 20,
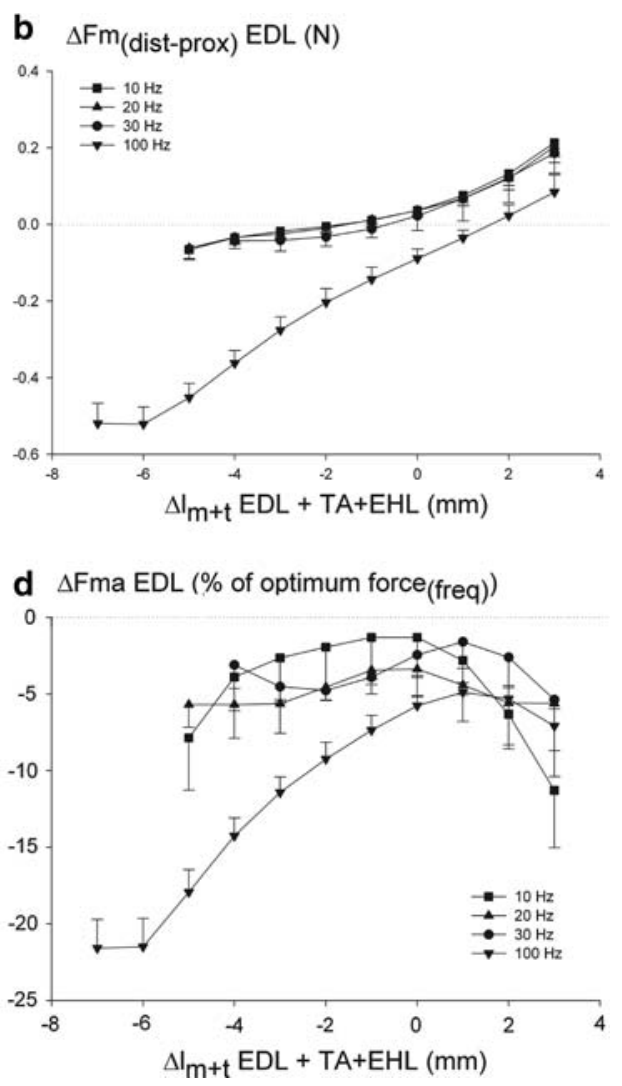

calculated by subtracting proximal total force from distal total force for any given muscle length. The negative value for the EDL proximo-distal difference in force indicates the presence of a net distally directed load exerted onto EDL, a positive value indicates a proximally directed net myofascial load. Values are shown as mean $\pm \mathrm{SE}, n=6$. c EDL proximo-distal differences in active $\left(\Delta F_{\mathrm{ma}}\right)$ and passive $\left(\Delta F_{\mathrm{mp}}\right)$ forces for $10,20,30$ and $100 \mathrm{~Hz}$ stimulation, as a function of anterior crural muscle lengthening. Anterior crural muscle-tendon complex length is expressed as a deviation from EDL $100 \mathrm{~Hz}$ distal optimum length. Values are shown as mean $\pm \mathrm{SE}, n=6$. d EDL proximo-distal force differences in active forces, $\Delta F_{\mathrm{ma}}$, normalized for distal optimum active force per frequency as a function of anterior crural muscle length. Values are shown as mean $\pm \mathrm{SE}, n=6$

30 and $100 \mathrm{~Hz}$, EDL optimum length shifted to lower muscle lengths as firing frequency was increased (e.g. for $100 \mathrm{~Hz}$, the shift in optimum muscle length equaled approximately $3 \mathrm{~mm}$ with respect to $20 \mathrm{~Hz}$ ).

Also for EDL $10 \mathrm{~Hz}$ active force deviating patterns were observed, but they were smaller than effects for TA + EHL: (a) EDL optimum muscle length for $10 \mathrm{~Hz}$ could not be shown to differ significantly from that at 20 and $30 \mathrm{~Hz}$, but differed significantly from $100 \mathrm{~Hz}$ optimum length. (b) For a considerable length range (i.e. $\left.5<\Delta l_{m+t}<+1\right) 10$ and $20 \mathrm{~Hz}$ forces were quite similar. These two results combined also indicate for EDL that $10 \mathrm{~Hz}$ forces are enhanced in some way over a considerable length range. 
Distal active slack lengths were shown (ANOVA) to differ significantly as a function of firing frequency. However, Bonferroni post-hoc tests showed that distal active slack length between the three submaximal stimulation frequencies did not differ significantly.

\section{Effects of stimulation frequency on EDL proximo-distal force differences}

The presence of any difference in proximally and distally measured forces is indicative of epimuscular myofascial force transmission. A negative value for this difference indicates a net myofascial loading of EDL in distal direction. This additional load is resisted by EDL force and the net load is integrated into the force exerted at the proximal tendon, causing proximally measured force to be higher than distally measured force.

The proximo-distal differences in total EDL force $\left(\Delta F_{\mathrm{m}}\right)$ represent the net myofascial load exerted on EDL during activation (Fig. 5b). ANOVA showed effects of anterior crural muscle length and firing frequency, as well as an interaction. Bonferroni post-hoc tests showed that $\Delta F_{\mathrm{m}}$ is not significantly different for the submaximal firing frequencies. In addition, $\Delta F_{\mathrm{m}}$ at $100 \mathrm{~Hz}$ stimulation differs significantly from $\Delta F_{\mathrm{m}}$ at the submaximal firing frequencies. Initially, at the beginning of the experiment, $\Delta F_{\mathrm{m}}$ attains negative values for submaximally as well as maximally active EDL, which indicates that a net total myofascial load is exerted on EDL in the distal direction. For the submaximal firing frequencies, this distally directed myofascial load is small, whereas the distally directed load on maximally active EDL is substantial $(0.5 \mathrm{~N})$. At $\Delta l_{m+t} \geq-1$, the net myofascial load exerted at submaximally active EDL is reversed as the $\Delta F_{\mathrm{m}}$ becomes positive and increases with increasing anterior crural muscle length. However, for maximally active EDL, $\Delta F_{\mathrm{m}}$ initially decreases with increasing anterior crural muscle length, only to become positive at $\Delta l_{m+t}>1$. Note that at high muscle lengths, the proximally directed myofascial loads are largest for the submaximal firing frequencies $(0.2 \mathrm{~N})$.

The proximo-distal differences in total EDL force are unequivocal evidence for the exertion of myofascially transmitted loads on maximally, as well as submaximally active EDL.

To allow a more thorough analysis of the contributions of the muscles' active and passive forces, the myofascial load exerted on EDL is analyzed for active and passive condition (Fig. 5c). Note however that proximo-distal differences in EDL active force can at best be only estimated, as active force is not measured directly, but calculated by subtracting passive force (measured before the tetanic plateau) from total force (measured during the tetanic plateau) (MacIntosh and MacNaughton 2005).
For all muscle lengths studied, EDL proximo-distal differences in active force $\left(\Delta F_{\mathrm{ma}}\right)$ are negative (Fig. 5c). Bonferroni post-hoc tests showed that $\Delta F_{\text {ma }}$ at the submaximal stimulation frequencies differs significantly from that at $100 \mathrm{~Hz}$ stimulation, but no significant difference in $\Delta F_{\text {ma }}$ is found between the submaximal firing frequencies. $\Delta F_{\text {ma }}$ for 10,20 and $30 \mathrm{~Hz}$ is quite small $\left(\Delta F_{\text {ma }}=0.03 \mathrm{~N}\right.$ or $5 \%$ of $\left.F_{\text {mao }}\right)$. Note, however, that this value is significantly different from zero (one sample $t$ test on average values). In addition, no significant effect of anterior crural muscular length could be shown on $\Delta F_{\text {ma }}$ at submaximal firing frequencies.

In contrast, for $100 \mathrm{~Hz}$ firing frequency, the $\Delta F_{\mathrm{ma}}$, and thus the net active myofascial load, is length-dependent and is highest at low muscle lengths but decreases after anterior crural muscles are lengthened distally. Note that at the lowest lengths, this value represents all of the active force exerted by EDL.

The normalized proximo-distal active force differences (i.e. $\Delta F_{\text {ma }}$ for each frequency normalized for distal EDL optimum force at that frequency) represent the fraction of EDL active force transmitted myofascially (Fig. 5d). For this variable, ANOVA showed effects of length, as well as of firing frequency, but no interaction. Bonferroni post-hoc tests showed that normalized $\Delta F_{\text {ma }}$ for maximal stimulation is significantly different from the submaximal stimulation frequencies; particularly at low muscle lengths, normalized $\Delta F_{\mathrm{ma}}$ for $100 \mathrm{~Hz}$ is significantly higher than for the submaximal firing frequencies. No significant differences between the three submaximal firing frequencies could be shown.

For the absolute proximo-distal differences in passive EDL force, ANOVA showed significant effects of anterior crural muscle length and of stimulation frequency on the EDL proximo-distal active force difference, but no significant interaction effect was shown.

\section{Discussion}

Extramuscular myofascial force transmission between antagonistic muscle groups

The main two findings of this study are:

1. The presence of significant mechanical interaction between, maximally, as well as submaximally excited, agonistic and antagonistic muscle groups.

2. The presence of, effects of firing frequencies on this interaction. These effects are similar or somewhat smaller for muscle group lengthening compared to those of single muscle lengthening (Meijer et al. 2006). However, as firing frequency is lowered, and active 
force exerted at the triceps surae and peroneal distal tendons decreases, the fraction of the force transmitted via extramuscular myofascial connections is equal to or even higher than for maximal stimulation (Figs. 2b, $3 b)$. Therefore, the conclusion is warranted that mechanical interaction between antagonistic muscles via extramuscular myofascial force transmission is substantial, even at submaximal firing frequencies. Such importance of myofascial force transmission at submaximal stimulation frequencies is in agreement with previous conclusions regarding myofascial force transmission between rat synergistic EDL and $\mathrm{TA}+\mathrm{EHL}$ for distal lengthening of EDL exclusively (Meijer et al. 2006).

Rat peroneal muscles are located within a compartment adjacent to the anterior crural one, and separated from it by the anterior intermuscular septum (Fig. 6). A very small part of the lateral gastrocnemius muscle is adjacent to the anterior crural and peroneal compartments, but the major part of the triceps surae muscle mass is located at the opposite site of the anterior crural compartment within the lower leg (Fig. 6). All connections between anterior crural

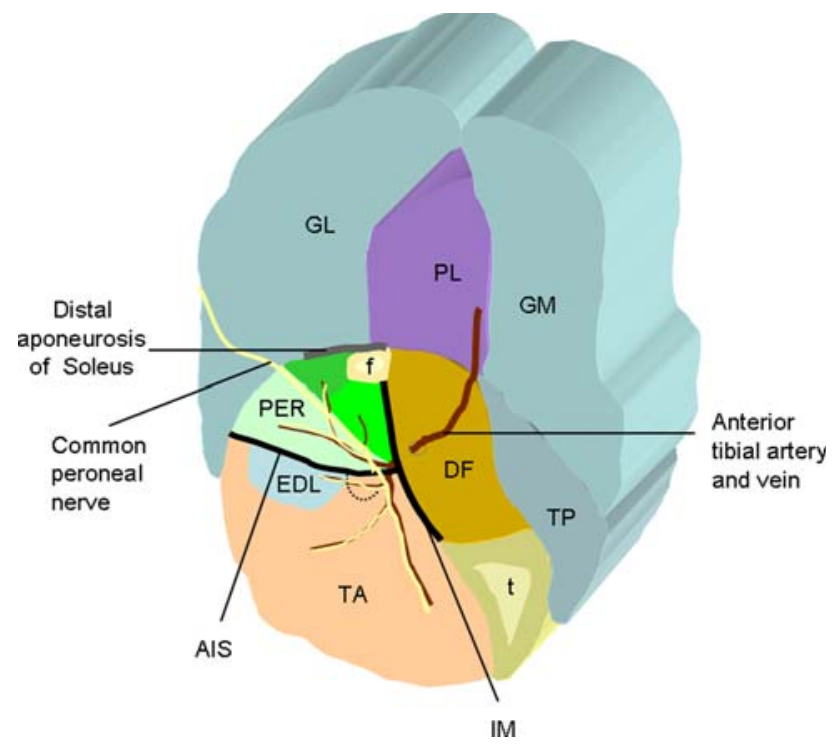

Fig. 6 Schematic representation of a cross-section of the rat lower leg with the biceps femoris muscle removed. Most of the crosssection represents the anatomy at approximately $1 / 3$ of the length of the EDL muscle belly from its origin. Note that the course of the common peroneal nerve and major blood vessels (anterior tibial artery and vein) is projected upon the cross-section from different levels. The EHL is only present more distally, but its position with respect to EDL and TA + EHL is indicated by a dotted line. Of the soleus muscle (SOL), only the distal aponeurosis is visible yet. GL lateral gastrocnemius muscle, $G M$ medial gastrocnemius muscle, $P L$ plantaris muscle, $P E R$ peroneal muscles, $D F$ deep flexor muscles, $T P$ tibialis posterior muscle, $f$ fibula, $t$ tibia, $I M$ interosseal membrane and $A I S$ anterior intermuscular septum and antagonistic peroneal and triceps surae muscles are extramuscular myofascial connections, such as compartmental fasciae, the neurovascular tract or epitendinous tissues. Via these extramuscular myofascial connections, force is transmitted from the peroneal and triceps surae muscles onto other muscles within the same or other compartments, joint capsules and ligaments, and ultimately bones. For maximally stimulated muscles, such transmission is in agreement with previous studies on extramuscular myofascial force transmission between antagonists (Huijing et al. 2007; Rijkelijkhuizen et al. 2007). In fact, such transmission has been shown for conditions of maximal activation in a combination of studies for all rat lower hind leg muscle groups (anterior crural, peroneal, deep flexors, and triceps surae (Huijing 2007; Meijer et al. 2007).

It should be noted that in our current and previous experiments, the length and position of only one muscle or muscle group were changed simultaneously, with all other muscles kept at constant muscle- tendon complex lengths to be able to unequivocally demonstrate the myofascial effects. However, in vivo movement will involve simultaneous and opposite length changes of antagonistic muscle groups (particularly anterior crural and triceps surae muscles). Therefore, changes in relative position of these antagonistic muscles will be more pronounced. As a consequence, it is expected that in vivo, myofascial force transmission between submaximally active antagonistic muscle groups will even attain higher maximal values than in our present work.

A fraction of the force transmitted from peroneal and triceps surae muscles is exerted onto anterior crural muscles via extramuscular pathways. Potential extramuscular myofascial pathways between anterior crural muscles and triceps surae and peroneal muscles are judged to be the collagenous stromata that reinforce the blood vessels and nerves of the lower leg (Fig. 6). Such complexes are referred to as the neurovascular tract. Within the rat lower leg, the neurovascular tract connects the anterior crural compartment and triceps surae and peroneal compartments in two ways:

1. the neurovascular tract itself forms a direct connection between antagonistic muscles, as the anterior tibial artery and vein descend from the triceps surae compartment via the deep flexor compartment into the peroneal and anterior crural compartments. Here they are joined by the deep peroneal nerve, entering from the peroneal into the anterior crural compartment.

2. via the attachment of the neurovascular tract to both sides of the anterior intermuscular septum, as well as to the epimysia of individual muscles and general fascia bordering all compartments. Distal lengthening 
of the anterior crural muscles will shear the neurovascular tract and results in stiffer extramuscular myofascial connections between peroneal and triceps surae and anterior crural muscles. Maas et al. (2005) suggested that for rat muscles within the anterior crural compartment, this extramuscular collagen-reinforced system is the major contributor to epimuscular myofascial force transmission between synergistic muscles. Also for humans, the neurovascular tract is thought to be a pathway for force transmission, providing a possible pathway for myofascial transmission between triceps surae and deep flexor compartments: Huijing (2007) reports indications of substantial loading of the human neurovascular tract (containing the tibial nerve in the popliteal cavity of the knee), as a result of simultaneous extension of the hip and knee and dorsal flexion of the ankle. Our current work implies that the neurovascular tract is also an important candidate pathway for intercompartmental myofascial force transmission between multiple antagonistic compartments in the rat lower limb.

Extramuscular myofascial force transmission and deviating length-force characteristics of EDL and $\mathrm{TA}+\mathrm{EHL}$

Any proximo-distal difference in force is taken as an indication for epimuscular myofascial force transmission (e.g. Huijing and Baan 2001a, b; Huijing et al. 1998; Maas et al. 2001). For submaximal firing frequencies, this difference in EDL forces is small (Fig. 5c), possibly suggesting that the effects of epimuscular myofascial force transmission on EDL may be negligible. Also, in contrast to earlier work on the effects of length of submaximally active EDL (Meijer et al. 2006), an increase in the fraction of the force transmitted myofascially with lower firing frequencies, indicative of an enhanced importance of myofascial force transmission at low firing frequencies, is absent (Fig. 5d). However, it should be kept in mind that the proximo-distal force difference represents only the net result of oppositely directed myofascial loads (Huijing 2007; Meijer et al. 2007). This becomes particularly evident when considering the total proximo-distal difference (Fig. 5b) at high muscle lengths; although the net total myofascial load on EDL is proximally directed, the separate active and passive myofascial loads on EDL are oppositely (i.e. distally and proximally respectively) directed (Fig. 5c). Therefore, a small proximo-distal force difference may actually represent multiple large myofascial loads being exerted on the muscle. Such conditions should have significant consequences for length-force characteristics. As our present results contain such effects, we argue that despite the small proximo-distal EDL active force differences, large extramuscular myofascial loads are exerted on anterior crural muscles. This is most evident for $10 \mathrm{~Hz}$ firing frequency, for which TA + EHL and EDL length-distal force characteristics deviate in a major way from expectations: with $10 \mathrm{~Hz}$ force exceeding that of 20 and even $30 \mathrm{~Hz}$ force at lower lengths. Also, the $10 \mathrm{~Hz}$ length- curves at higher lengths do not fit predicted effects of firing frequency described for either fully dissected muscles (i.e. with only the nerve and blood supply left intact) or single muscle fibers. Work on fully dissected muscle (Rack and Westbury (1969) on cat m. soleus; Roszek and Huijing (1997) on rat m. gastrocnemius; Brown et al. (1999) on cat caudofemoralis) and on skinned single muscle fibers (Stephenson and Wendt 1984) did show that as stimulation frequency or concentration of $\mathrm{Ca}^{++}$is lowered, optimum length and, for dissected muscle also active slack length, shift progressively to higher muscle lengths. In agreement with previous work from our laboratory (Meijer et al. 2006) we conclude that for a muscle within an intact compartment, intrinsic properties and mechanisms alone do not suffice to explain firing frequency-related changes of length-force characteristics, because myofascial loads are integrated into the force exerted at the tendons. It should be noted that to explain higher EDL forces exerted distally for $10 \mathrm{~Hz}$, a proximally directed load has to be exerted near to the distal end of the EDL and TA + EHL muscle-tendon complexes. The contrast between such a load and the observed net distally directed load illustrates the complexity of myofascial loading of muscle. In the present experimental conditions, proximally directed myofascial loads are expected to be exerted on anterior crural muscles by peroneal and triceps surae muscles via the neurovascular tracts. For conditions of maximal activation, it was recently shown (Huijing 2007) that a distal load is a common feature of a distally shortened muscle.

In human patients suffering from spastic paresis, such load becomes very apparent after tenotomy of the flexor carpi ulnaris (FCU) muscle. As the wrist is moved from palmar flexion, the tenotomized passive or active FCU is lengthened, despite the fact that FCU no longer crosses the wrist (Kreulen et al. 2003; Smeulders and Kreulen 2007). These results indicate that extending the wrist causes a distally directed force to be exerted onto FCU, presumably by straining of the neurovascular tract or other myofascial connections.

For the rat, a distally directed load is imposed on the anterior crural muscles by the distal segments of compartmental and general fasciae of the anterior crural compartment. These fasciae attach to the tibial crest, run laterally over the TA muscle belly, attach to its epimysium and continue towards to the antagonistic muscles (Meijer 
et al. 2007). A fraction of this distally directed myofascial load exerted on TA + EHL is exerted onto EDL via epimuscular myofascial connections between EDL and TA + EHL. Distal lengthening of all anterior crural muscles decreases the distally directed load exerted onto EDL (Meijer et al. 2007). At low firing frequencies, for any given muscle length, lower forces are exerted at the serieselastic structures, i.e. the myotendinal and the myofascial pathways, and consequently the force transmitted via these pathways may be reduced. It should be realized that imposing such loads at different locations along the muscle fibers and variable locations within the muscle will lead to (enhanced) distributions serial and parallel distribution in sarcomere lengths (Yucesoy et al. 2003).

In conclusion, for rat muscles active within their natural context of connective tissues, mechanical interaction between antagonistic muscles via extramuscular myofascial pathways is also substantial at submaximal stimulation frequencies. Antagonistic muscles need not be adjacent for such interaction. They interact even if located at opposite sites of the lower leg. Such extramuscular myofascial force transmission between antagonistic compartments significantly modifies the effects of firing frequency on muscle length-force characteristics. Further work on human subjects is indicated to evaluate such effects in different conditions of natural movement during health and disease.

Open Access This article is distributed under the terms of the Creative Commons Attribution Noncommercial License which permits any noncommercial use, distribution, and reproduction in any medium, provided the original author(s) and source are credited.

\section{References}

Bloch RJ, Gonzalez-Serratos H (2003) Lateral force transmission across costameres in skeletal muscle. Exerc Sport Sci Rev 31:73-78

Brown IE, Cheng EJ, Loeb GE (1999) Measured and modeled properties of mammalian skeletal muscle II. The effects of stimulus frequency on force-length and force-velocity relationships. J Muscle Res Cell Motil 20:627-643

Hennig R, Lomo T (1985) Firing patterns of motor units in normal rats. Nature 314:164-166

Huijing PA (2003) Muscular force transmission necessitates a multilevel integrative approach to the analysis of function of skeletal muscle. Exerc Sport Sci Rev 31:167-175

Huijing PA (2007) Epimuscular myofascial force transmission between antagonistic and synergistic muscles can explain movement limitation in spastic paresis. J Electromyogr Kinesiol 17:708-724

Huijing PA, Baan GC (2001a) Extramuscular myofascial force transmission within the rat anterior tibial compartment: proximodistal differences in muscle force. Acta Physiol Scand 173:297311

Huijing PA, Baan GC (2001b) Myofascial force transmission causes interaction between adjacent muscles and connective tissue: effects of blunt dissection and compartmental fasciotomy on length-force characteristics of rat extensor digitorum longus muscle. Arch Physiol Biochem 109:97-109

Huijing PA, Baan GC (2003) Myofascial force transmission: muscle relative position and length determine agonist and synergist muscle force. J Appl Physiol 94:1092-1107

Huijing PA, Baan GC, Rebel GT (1998) Non-myotendinous force transmission in rat extensor digitorum longus muscle. J Exp Biol 201:682-691

Huijing PA, Jaspers RT (2005) Adaptation of muscle size and myofascial force transmission: a review and some new experimental results. Scand J Med Sci Sports 15:349-380

Huijing PA, van de Langenberg RW, Meesters J, Baan GC (2007) Extramuscular myofascial force transmission also occurs between synergistic muscles and antagonistic muscles. J Electromyogr Kinesiol 17:680-689

Kreulen M, Smeulders MJ, Hage JJ, Huijing PA (2003) Biomechanical effects of dissecting flexor carpi ulnaris. J Bone Joint Surg Br 85:856-859

Maas H, Baan GC, Huijing PA (2001) Intermuscular interaction via myofascial force transmission: effects of tibialis anterior and extensor hallucis longus length on force transmission from rat extensor digitorum longus muscle. J Biomech 34:927-940

Maas H, Baan GC, Huijing PA, Yucesoy CA, Koopman BH, Grootenboer HJ (2003a) The relative position of EDL muscle affects the length of sarcomeres within muscle fibers: experimental results and finite-element modeling. J Biomech Eng 125:745-753

Maas H, Yucesoy CA, Baan GC, Huijing PA (2003b) Implications of muscle relative position as a co-determinant of isometric muscle force: a review and some experimental results. J Mech Med Biol 3:145-168

Maas H, Meijer HJM, Huijing P (2005) Intermuscular interaction between synergists in rat originates from both intermuscular and extramuscular myofascial force transmission. Cells Tissues Organs 181:38-50

MacIntosh BR, MacNaughton MB (2005) The length-dependence of muscle active force: considerations for parallel elastic properties. J Appl Physiol 98:1666-1673

Meijer HJM, Baan GC, Huijing PA (2006) Myofascial force transmission is increasingly important at lower forces: firing frequency-related length-force characteristics of rat extensor digitorum longus. Acta Physiol (Oxf) 186:185-195

Meijer HJM, Rijkelijkhuizen JM, Huijing PA (2007) Myofascial force transmission between antagonistic rat lower limb muscles: effects of single muscle or muscle group lengthening. J Electromyogr Kinesiol 17:698-707

Monti RJ, Roy RR, Hodgson JA, Edgerton VR (1999) Transmission of forces within mammalian skeletal muscles. J Biomech $32: 371-380$

Neter J, Wasserman W, Kutner ME (1990) Applied linear statistic models: regression. analysis of variance and experimental design, Irwin, Homewood

Pasternak C, Wong S, Elson E (1995) Mechanical function of dystrophin in muscle cells. J Cell Biol 128:355-361

Rack PM, Westbury DR (1969) The effects of length and stimulus rate on tension in the isometric cat soleus muscle. J Physiol (Lond) 204:443-460

Rijkelijkhuizen JM, Baan GC, de Haan A, de Ruiter CJ, Huijing PA (2005) Extramuscular myofascial force transmission for in situ rat medial gastrocnemius and plantaris muscles in progressive stages of dissection. J Exp Biol 208:129-140

Rijkelijkhuizen JM, Meijer HJM, Baan GC, Huijing PA (2007) Myofascial force transmission also occurs between antagonistic muscles located within opposite compartments of the rat lower hind limb. J Electromyogr Kinesiol 17:690-697 
Roszek B, Huijing PA (1997) Stimulation frequency history alters length-force characteristics of fully recruited rat muscle. J Electromyogr Kinesiol 7:161-177

Smeulders MJ, Kreulen M (2007) Myofascial force transmission and tendon transfer for patients suffering from spastic paresis: A review and some new observations. J Electromyogr Kinesiol 17:644-656

Stephenson DG, Wendt IR (1984) Length dependence of changes in sarcoplasmic calcium concentration and myofibrillar calcium sensitivity in striated muscle fibers. J Muscle Res Cell Motil 5:243-272

Street SF (1983) Lateral transmission of tension in frog myofibers: a myofibrillar network and transverse cytoskeletal connections are possible transmitters. J Cell Physiol 114:346-364

Street SF, Ramsey RW (1965) Sarcolemma: transmitter of active tension in frog skeletal muscle. Science 149:1379-1380
Sheard P, Paul A, Duxson M (2002) Intramuscular force transmission. Adv Exp Med Biol 508:495-499

Tidball JG (1991) Force transmission across muscle cell membranes. J Biomech 24:43-52

Trotter JA, Hsi K, Samora A, Wofsy C (1985) A morphometric analysis of the muscle-tendon junction. Anat Rec 213:26-32

Yucesoy CA, Koopman BH, Baan GC, Grootenboer HJ, Huijing PA (2003) Effects of inter- and extramuscular myofascial force transmission on adjacent synergistic muscles: assessment by experiments and finite-element modeling. J Biomech 36:17971811

Yucesoy CA, Maas H, Koopman BH, Grootenboer HJ, Huijing PA (2006) Mechanisms causing effects of muscle position on proximo-distal muscle force differences in extra-muscular myofascial force transmission. Med Eng Phys 28:214-226 